\title{
Analysis of Urea in Petfood Matrices: Comparison of Spectro-Colorimetric, Enzymatic and Liquid Chromatography Electrospray Ionization High Resolution Mass Spectrometry Methods
}

\author{
Patrick Pibarot $^{1}$, Serge Pilard ${ }^{2}$ \\ ${ }^{1}$ Nestlé Research and Development Centers, Aubigny, France \\ ${ }^{2}$ Analytical Platform, University of Picardie Jules Verne, Amiens, France \\ Email: Patrick.Pibarot@rdam.nestle.com
}

Received July 4, 2012; revised August 12, 2012; accepted August 19, 2012

\begin{abstract}
Adulteration may consist in non authorized source of nitrogen addition to increase the protein content of some raw materials. Urea which is authorized for feed is a non nutritional source of nitrogen in food and pet food. Adulteration of food or pet food raw material by urea is thus monitored by manufacturer and governmental authorities with official methods which are either enzymatic (Association of Official Agricultural Chemists, AOAC) or spectro-colorimetric (European Community, EC). Each method gives results which are not comparable and spectro-colorimetric methods may result in false-positive urea detection. Liquid chromatographic (LC/UV-DAD) analysis of extracts from spectrocolorimetric method indicates that presence of free amino-acid may interfere with colorimetric detection of urea in the EC method with pet food samples. Liquid chromatography electrospray ionization high resolution mass spectrometry (LC/ESI-HRMS) has allowed to quantify low content $(<0.01 \%)$ of urea in pet food water extracts for samples which resulted in significant urea detection with colorimetric method and in content below the detection threshold with enzymatic method. This study demonstrates the EC colorimetric method is not applicable to pet food and also food samples which have a complex composition with significant levels of free amino acids. On the other hand we clearly evidenced by means of the LC/ESI-HRMS results that the AOC Enzymatic method is applicable to urea quantification in pet food samples and gives reliable results.
\end{abstract}

Keywords: Pet Food; Food; Urea; Adulteration; Enzymatic Method; Spectro-Colorimetry; LC/UV-DAD; LC/ESI-HRMS

\section{Introduction}

Non authorized food ingredient can be used to adulterate ingredients, raw materials (e.g. Gluten) or finished product (e.g. milk products) to artificially increase the protein content. Melamine, for instance, has been used as the food additive to increase the apparent protein content in food products [1].

Ruminant can convert non protein nitrogen into protein and urea may be used to increase the nutritional nitrogen content of feed, hay or silage. Urea is authorized as a component of animal feeds to provide nutritional source of nitrogen. Urea is accepted as a "Generally Recognized as Safe" food additive and is also used as a fermentation aid in foods and beverages [2].

Urea is produced in the liver of mammals as an endproduct of protein metabolism and excreted in urine. Humans excrete up to 30 grams of urea per day in the urine [2].
Avian species (e.g. poultry) metabolize mainly uric acid $(55 \%-72 \%)$ and some urea in lower proportion (2\% $11 \%)$, the rest of protein metabolism product is ammonia $(11 \%-21 \%)[3,4]$.

Therefore, poultry meat and by products should not present significant amount of urea while mammal meat by-product may contain some urea in more significant amounts.

Kjeldahl or Dumas methods measure the protein content by analyzing total nitrogen content, without identifying its sources. The research of non-protein nitrogen source is complementary to protein analysis to check the absence of adulteration in raw materials and finished products.

Urea is one possible molecule which may be used to adulterate (increase nitrogen content) food and petfood or one of the ingredients used to manufacture food or petfood. 
Therefore, even if toxicology is not an issue, the analysis of urea in food ingredients and in finished products may be of importance to prevent the use of non authorized nitrogen sources in food and petfood industries.

Various analytical methods including colorimetry, urea enzymatic conversion to ammonia, liquid or gas chromatography or near infrared spectrometry are described for urea analysis in feed, silage, fertilizers, blood, serum, urine and other matrices [5].

The two main analytical methods used in food and feed areas are:

- Spectro-colorimetric methods [6,7];

- Enzymatic (urease) methods [8].

These methods are either based:

- On the reaction of urea with a specific molecule to create a chromophore which content is analyzed by spectro-colorimetry;

- Or on the enzymatic degradation of urea in presence of urease to ammonia and on ammonia analysis by the Kjeldahl method (acidic titration) or other various methods.

Their main advantage is rapid and easy to use protocol.

Other more sophisticated methods based on gas chromatography coupled to isotope ratio [9] or isotope dilution $[10,11]$ mass spectrometry and liquid chromatography hyphenated to tandem mass spectrometry [1] have been developed.

Several cases of interference in colorimetric method or enzyme activity disturbance are reported $[5,11,12]$.

Co-elution problem leading to result variations in chromatography methods are also reported [13].

The objective of the current study is:

- To compare the results of spectro-colorimetric method and enzymatic method reliability for the routine analysis of urea dry petfood products;

- To define which method is the most suitable for urea analysis in dry petfood by comparing the results obtained by the routine methods to the results obtained with liquid chromatography electrospray ionization high resolution mass spectrometry (LC/ESI-HRMS).

Finally the conclusion of the study should allow to recommend an official method for analyzing urea in dry petfood products and in pet food raw materials.

\section{Experimental Sections}

\subsection{Spectro-Colorimetric Method (European Regulation)}

This method corresponds to the method recommended by the European Union and is described in European Commission Regulation (EC) No. 152/2009 laying down the methods of sampling and analysis for the official control of feed [6]. This method is also described as a AOAC method [7].

The sample is suspended in water with a clarifying agent (active carbon). The suspension is filtered. The urea content of the filtrate is determined after the addition of 4dimethylaminobenzaldehyde (4-DMAB) by measuring the optical density at a wavelength of $420 \mathrm{~nm}$. If the sample contains simple nitrogenous compounds such as amino acids, the optical density shall be measured at $435 \mathrm{~nm}$.

\subsection{Enzymatic Method}

The enzymatic method is the official method of urea analysis in feed of AOAC international (No. 941.04) [8].

The method consists in digesting $1 \mathrm{~g}$ of samples in urease enzyme solution at $\mathrm{pH}=7$ in order to convert the urea in carbon dioxide and ammonia. After alkalization, ammonia vapor is released and distillated in a known amount of acid and the distillate titrated with standard base solution (Kjedhal method).

It should be noticed that ammonia is analyzed together with urea and that some heavy metal may inhibit the activity of urease.

\subsection{Liquid Chromatography Ultraviolet-Visible Diode Array Detection (LC/UV-DAD)}

The analyses were run on AS DX 500 HPLC (Dionex, Sunnyvale CA, USA) equipment with UV-Vis Diode Array detector UVD $340 \mathrm{~S}$.

After several trials with several columns and elution conditions (ion exchange, $\mathrm{pH}$ adjustment) the following chromatographic conditions were selected. The reverse phase column was an Acclaim PA2 C18, $4.6 \times 150$ mm, $5 \mu \mathrm{m}$ supplied by Dionex France.

The chromatographic conditions were:

- Isocratic elution with $100 \%$ purified water at $1 \mathrm{~mL} / \mathrm{min}$ and $30^{\circ} \mathrm{C}$;

- Sample injection $250 \mu \mathrm{L}$ (auto-sampler AS 50);

- Detection 200 - 595 nm (resolution 1nm). Urea quantification is performed at $435 \mathrm{~nm}$ as indicated in the EC protocol [6,7].

The samples of petfood were ground and extracted in the same conditions than for the DMAB spectro-colorimetric analysis (except active carbon treatment). The extract reacted with DMAB were directly injected on the HPLC column. The separation and detection of the molecules produced by reaction between urea and DMAB has been chosen with two objectives:

- To improve the selectivity and sensibility of the urea detection by UV absorbance;

- To check the presence of other molecules than urea in dry pet food which may react with DMAB and absorb at the wavelength used by the EC colorimetric method.

\subsection{Liquid Chromatography Electrospray Ionization High Resolution Mass Spectrometry (LC/ESI-HRMS)}

The LC/ESI-HRMS experiments were performed on a Q- 
TOF Ultima Global hybrid quadrupole time-of-flight instrument (Waters-Micromass, Manchester, UK), coupled with an Ultra-Fast Liquid Chromatograph (UFLC) system (Shimadzu, Duisburg, Germany).

The separation was obtained on a Nucleodur Pyramid $2.0 \mathrm{~mm}$ i.d. $\times 250 \mathrm{~mm}, 3 \mu \mathrm{m} \mathrm{C}_{18}$ reversed phase column (Macherey-Nagel, Duren, Germany) at $25^{\circ} \mathrm{C}$, using an isocratic elution with $100 \%$ water. The flow-rate and the sample injection volume were set to $0.2 \mathrm{~mL} / \mathrm{min}$ and 10 $\mu \mathrm{L}$, respectively.

The effluent from the LC column was directly introduced in the electrospray ion source (Z-spray) and the ESI-HRMS data were recorded in the positive ion mode with capillary and cone voltages of $3.7 \mathrm{kV}$ and $184 \mathrm{~V}$, respectively. Nitrogen was used as a nebulizing $(100 \mathrm{~L} / \mathrm{h})$ and a drying $(450 \mathrm{~L} / \mathrm{h})$ gas. The source and desolvatation temperatures were kept at $120^{\circ} \mathrm{C}$ and $250^{\circ} \mathrm{C}$, respectively. The mass range was 100 - $200 \mathrm{Da}$ and spectra were recorded in the profile mode at a scan rate of $0.1 \mathrm{~s} / \mathrm{scan}$ (interscan time: $0.1 \mathrm{~s}$ ) and at a resolution of 5,000 (FWHM). Accurate mass calibration was achieved using the cluster ions of an orthophosphoric acid solution $(0.2 \%$ in $\mathrm{H}_{2} \mathrm{O} / \mathrm{CH}_{3} \mathrm{CN} 50 / 50 \mathrm{v} / \mathrm{v}$ ). Data acquisition and processing were performed with MassLynx 4.0 SP4 software.

The ground pet food samples were extracted by mixing with water $\left(2 \mathrm{~g}\right.$ in $\left.500 \mathrm{~mL}, 30^{\circ} \mathrm{C}, 30 \mathrm{~min}\right)$ and sampling of the supernatant after decantation. Urea quantification was performed using the integration of the reconstructed ionic current (RIC) of the [di-Urea+Na] ${ }^{+}$ion $\left(\mathrm{C}_{2} \mathrm{O}_{2} \mathrm{H}_{8} \mathrm{~N}_{4} \mathrm{Na}\right.$, exact mass: 143.0545$)$ selected with a 150 ppm (0.02 Da) mass window. For each set of samples, urea calibration curves were obtained after injection of standard solutions prepared in water.

\section{Results and Discussion}

\subsection{Analyses of Dry Pet Food Finished Products by EC and AOAC Methods}

The DMAB spectro-colorimetric method is the European official method for analyzing urea in feed materials while in the American zone, for instance, the enzymatic method is the official one. The comparison of the two methods with a range of dry pet-food sample (from various manufacturers) was therefore an interesting exercise.

The results of this comparison are given in Table 1.

The result demonstrate the two methods do not give similar results and the spectro-colorimetric method gives systematically urea concentration between 0.14 and 0.27 $\mathrm{g} / 100 \mathrm{~g}$ while the enzymatic method gives non detectable amount of urea whatever is the analyzed sample (except one sample at $0.01 \mathrm{~g} / 100 \mathrm{~g}$ ).

Therefore either the enzymatic method is not able to detect urea in petfood samples (e.g. urease inhibition) or the spectro-colorimetric method results in false positive by detecting other interfering molecules than urea.
Table 1. Comparison of urea content (g/100g) obtained in dry pet food samples with spectro-colorimetric method and enzymatic method.

\begin{tabular}{ccc}
\hline Samples & EC colorimetric method & AOAC enzymatic method \\
\hline Dog food 1 & 0.18 & $<0.01$ \\
Dog food 2 & 0.14 & $<0.01$ \\
Dog food 3 & 0.26 & $<0.01$ \\
Dog food 4 & 0.25 & $<0.01$ \\
Cat food 1 & 0.26 & $<0.01$ \\
Cat food 2 & 0.25 & 0.01 \\
Cat food 3 & 0.27 & $<0.01$ \\
Cat food 4 & 0.17 & $<0.01$ \\
\hline
\end{tabular}

\subsection{Analysis of Raw Materials by EC and AOAC Methods}

If the finished product contains some non acceptable level of urea, some urea at higher level of concentration should be found in the raw materials used in the formulation of the finished products.

A wide range of raw materials have been analyzed. Significant concentrations of urea are found by the spectro-colorimetric method for animal and fish meals, viscera and liver digest, colostrum and pure-amino acids. All these colorimetric method positive samples are rich in free amino acids.

The highest concentrations are found with pure aminoacids which are already identified as molecules able to react with DMAB and to be detected by UV absorption even if the wavelength of $435 \mathrm{~nm}$ is selected by method authors to minimize the interference of amino-acids.

A selection of the ingredients with significant urea concentration determined by spectro-colorimetry was controled by enzymatic method. The results are shown in the following tables.

Two batches of methionine, which may be added in relatively significant amount in the recipe for palatability purpose, were analyzed and the results of two different samples are presented on Table 2.

Methionine is detected by the EC colorimetric method as urea even if obviously the quantification is far from the true value. Enzymatic method is not able to produce ammonia with methionine as a substrate.

Analysis results comparison by both methods was also performed with chicken viscera digest and pork liver digest (See Table 3).

Chicken viscera digest contains free amino acid (because of the enzymatic digestion) but is unlikely to contain significant amount of urea as chicken metabolism produces very limited amount of urea and mainly produces uric acid.

As matter of fact, the colorimetric method gives a positive answer while the enzymatic method does not detect any urea.

Pork liver digest contain higher level of free amino acid 
Table 2. Comparison of urea contents (g/100g) obtained with methionine ingredient samples by spectro-colorimetric (measured at $435 \mathrm{~nm}$ ) and enzymatic methods.

\begin{tabular}{ccc}
\hline & $\begin{array}{c}\text { Colorimetric method } \\
(\mathrm{g} / 100 \mathrm{~g})\end{array}$ & $\begin{array}{c}\text { Enzymatic method } \\
(\mathrm{g} / 100 \mathrm{~g})\end{array}$ \\
\cline { 2 - 3 } Methionin 1 & 5.56 & $<0.01$ \\
Methionin 2 & 4.67 & $<0.01$ \\
\hline
\end{tabular}

Table 3. Comparison of urea content (g/100g) obtained in chicken viscera digest with spectro-colorimetric and enzymatic methods.

\begin{tabular}{ccc}
\hline & $\begin{array}{c}\text { Colorimetric method } \\
(\mathrm{g} / 100 \mathrm{~g})\end{array}$ & $\begin{array}{c}\text { Enzymatic method } \\
(\mathrm{g} / 100 \mathrm{~g})\end{array}$ \\
\cline { 2 - 3 } Chicken viscera digest & 0.13 & $<0.01$ \\
Pork liver digest & 0.95 & 0.12 \\
\hline
\end{tabular}

and may contain urea as the pork metabolism (mammalian) produce urea. Again as a matter of fact, the colorimetric method results are higher than the enzymatic one because of the amino acid interferences and the enzymatic method is able to detect the natural low level of urea which is expected.

To make a further proof of the adequacy of the enzymatic method for the analysis of urea in petfood, pet food samples were spiked with 3 different levels of pure urea. Results are given in Table 4.

For the non spiked sample, the enzymatic method gave non detectable result. Sample spiked with known level of urea were correctly analyzed by the enzymatic method and this prove the enzymatic method is able to detect correctly urea when it is present in the pet food products.

This comparison of urea content results obtained by EC colorimetric method and AOAC enzymatic method, gives first indication that some interfering amino compound may result in false positive with the EC colorimetric method. This should be confirmed by analyzing the pet food samples with other analytical methods.

\subsection{LC/UV-DAD Analyses of DMAB Product Extracts}

The objective of the HPLC analysis of samples extracted and treated with DMAB in similar way than with the EC colorimetric method is to chromatographically separate the interfering compounds from urea in order to demonstrate that other molecules than urea are analyzed in pet food samples when EC colorimetric method is used.

Urea molecule presents low retention time with reverse or normal chromatography and is not easily separated from the free amino-acid or bio-amines [14]. After several trials the Acclaim PA2 C18 (Dionex) was selected as the most able to separate urea and free amino-acids. Four dry products from the super-premium cat and dog products range were selected for their significant contents in urea as analyzed with EC colorimetric method. These products were analyzed by HPLC after water extraction and reaction with DMAB as described in EC colorimetric protocol. Figure $\mathbf{1}$ gives an example of HPLC analysis with pet food sample spiked with $0.1 \%$ urea.

Analyses results of dog and cat pet food samples by HPLC method are given in Table 5.

Free amino acid peaks detected after the peak of urea (see Figure 1) were arbitrary quantified in order to rank them. It should be noticed that the detection was at 435 $\mathrm{nm}$ and only molecules able to react with DMAB were detected by the HPLC analysis.

The first fact is that the levels of urea quantified by the HPLC method are lower of a magnitude of about 100 to the content of urea quantified by the EC colorimetric method.

Nevertheless, no clear correlation is observed between either the levels of urea quantified by both methods neither between amino-acid arbitrary ranking and the urea levels.

Table 4. Results of analysis of spiked dry pet food by enzymatic method.

\begin{tabular}{cc}
\hline $\begin{array}{c}\text { Dry PF with spiked Urea (g/100g, } \\
\text { spike levels) }\end{array}$ & $\begin{array}{c}\text { Enzymatic method } \\
(\mathrm{g} / 100 \mathrm{~g})\end{array}$ \\
\hline 0 & $<0.01$ \\
0.5 & 0.57 \\
1 & 1.01 \\
1.5 & 1.38 \\
\hline
\end{tabular}

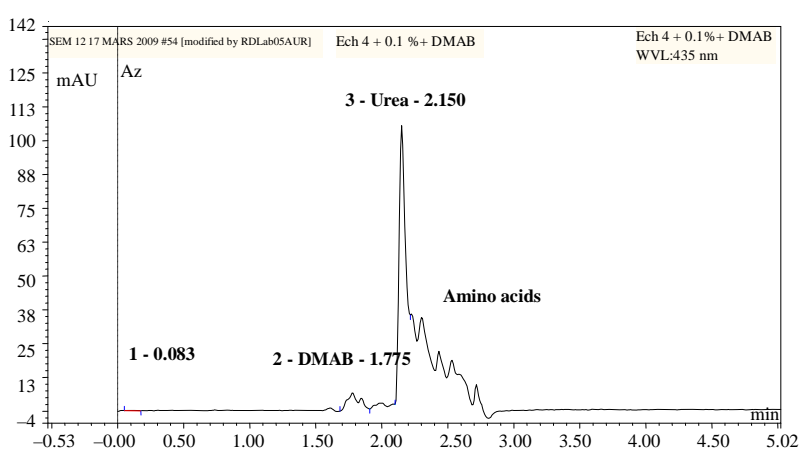

Figure 1. Example of chromatogram obtained by LC/UVDAD analysis of pet food sample spiked with $0.1 \%$ of urea (Acclaim C18, detection at $435 \mathrm{~nm}$, urea peak at $2.1 \mathrm{~min}$, DMAB at $1.7 \mathrm{~min}$ )

Table 5. Results of analysis of dry pet food by LC/UV-DAD, comparison with spectro colorimetric results.

\begin{tabular}{cccc}
\hline & Colorimetric method & \multicolumn{2}{c}{ LC/UV-DAD analysis } \\
\cline { 2 - 4 } Samples & Urea (g/100g) & Urea (g/100g) & $\begin{array}{c}\text { Amino acids } \\
\text { (arbitrary unit) }\end{array}$ \\
\hline Dog food 5 & 0.32 & 0.004 & 3.61 \\
Cat food 1 & 0.26 & 0.0058 & 5.99 \\
Cat food 5 & 0.60 & 0.0062 & 2.75 \\
Cat food 6 & 0.75 & 0.0038 & 3.99 \\
\hline
\end{tabular}


Therefore the LC/UV-DAD analyses have proven that other molecules than urea can react with DMAB and interfere in the colorimetric analysis to give overestimated results.

Even if some interesting facts are deduced from this HPLC analysis, the proof of the overestimation and the true level of urea in pet food samples is still to be done as the urea and amino-acid peaks separation is not enough to validate the quantification of urea. This lack of retention of urea and difficulty to separate urea from amino-acid in HPLC has been already observed by some authors [14].

The selectivity and the effective separation of urea with the other molecules derivatized by DMAB is not enough to seriously quantify the true level of urea in dry pet food.

\subsection{LC/ESI-HRMS of Pet Food Samples}

The objective of the LC/ESI-HRMS analysis was to access to urea content in pet food samples using a simple water extraction without any further reaction (e.g. DMAB) and a highly specific detection method. In order to separate as far as possible urea from the interfering molecules (e.g. free amino acids) we have used a liquid chromatography method developed by Macherey Nagel on a Nucleodur Pyramid $\mathrm{C}_{18}$ column with water (100\%) as the eluent [15], in these conditions urea was detected at a retention time of $2.86 \mathrm{~min}$.

Electrospray and atmospheric pressure chemical ionizations coupled to tandem mass spectrometry (ESI-MS/MS and APCI-MS/MS) were applied to determine the concentration of urea present in the water of swimming pool [16] but, to our knowledge, these methods were never used for urea quantification in complex samples, such as pet food. High resolution mass spectrometry, allowing the determination of molecule elemental composition, is a very selective and sensitive method to identify and quantify a specific compound in mixtures. Consequently, in these work we have investigated online LC/ESI-HRMS using the high resolution capabilities of a quadrupole orthogonal time-of-flight (Q-TFOF) mass spectrometer [17].

The exact mass which was used for urea characterization and quantification was 143.0545, corresponding to the elemental composition of the [di-Urea+Na] ${ }^{+}$ion $\left(\mathrm{C}_{2} \mathrm{O}_{2} \mathrm{H}_{8} \mathrm{~N}_{4} \mathrm{Na}\right)$. The mass selection window was set to $150 \mathrm{ppm}$ (0.02 Da) for reconstructed ion chromatogram (RIC of m/z 143.05 or 143.07 according to the mass spectrometer calibration).

Pure urea was diluted in water to prepare a range of standard solutions. A stock solution was prepared by dissolution of $40 \mathrm{mg}$ of pure urea in $1000 \mathrm{~mL}$ of de-ionized water to obtain a urea solution at $4 \mathrm{mg} / 100 \mathrm{~mL}$. The stock solution was diluted in water to prepare standard solutions at $0.2,0.4,0.8,1.2,1.6$ and $2 \mathrm{mg} / 100 \mathrm{~mL}$, in the range of the urea concentration expected in pet food samples. These standard solutions were injected in the LC/MS system to build a calibration curve before each analyses session. The chromatograms acquired at the exact mass of $143.05(\mathrm{~m} / \mathrm{z})$ and peak areas (retention time $2.86 \mathrm{~min}$.) obtained are presented on Figure 2.

The corresponding calibration curve was obtained from the least-squares linear regression presented with its correlation coefficient $\left(\mathrm{R}^{2}=0.9927\right)$ on Figure 3 .

The linearity of the calibration curve was demonstrated for urea concentrations up to $4 \mathrm{mg} / 100 \mathrm{~mL}$. For each dry pet food analysis $2 \mathrm{~g}$ of pellets were extracted in $500 \mathrm{~mL}$ pure water during 30 minutes at $30^{\circ} \mathrm{C}$. For each series of pet food samples new dilutions of urea stock solution at 4 $\mathrm{mg} / 100 \mathrm{~mL}$ were prepared to build a dedicated calibration curve.

\subsection{Pet Food Matrices Effect on LC/ESI-HRMS}

To validate the eventual effect of the pellet matrix on the quantification of urea by LC/ ESI-HRMS, the extraction of a urea doped dry pet food sample, which was previously analyzed by EC spectro-colorimetric method (result: $0.46 \mathrm{~g} / 100 \mathrm{~g}$ ), was performed. A sample of 4 grams of ground pellets was doped with respectively 40 and 20 milligrams of pure urea powder and the resulting samples analysis at 1\% urea (Chromatogram A) and 0.5\% (Chromatogram B) are shown Figure 4.

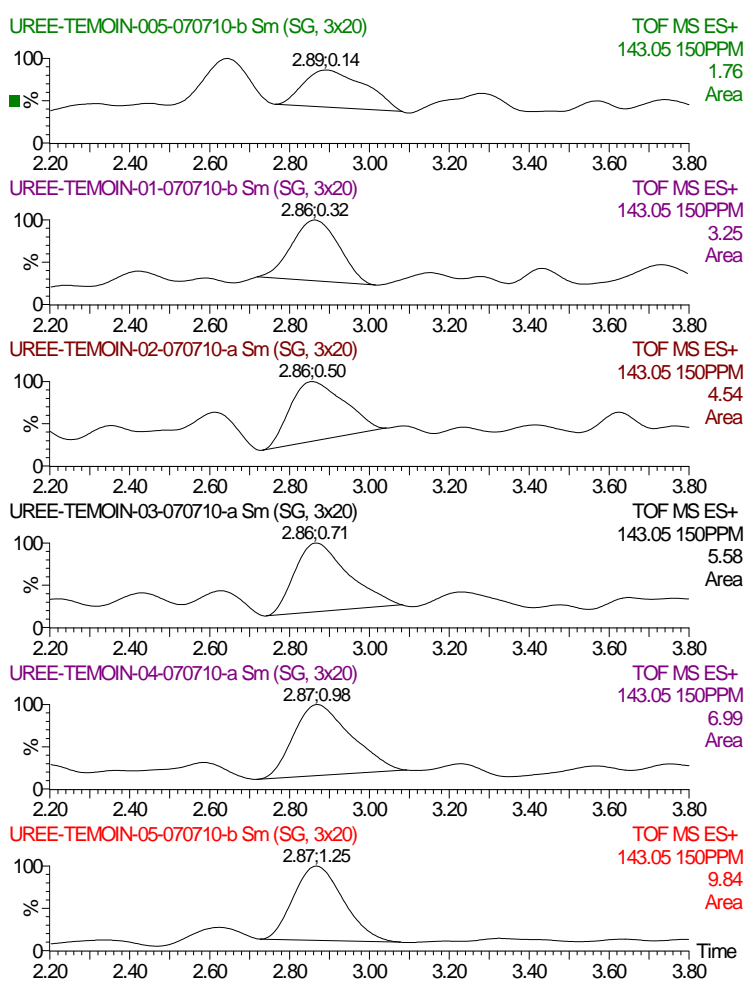

Figure 2. LC/ESI-HRMS analysis of urea standard solutions at $0.2,0.4,0.8,1.2,1.6$ and $2 \mathrm{mg} / 100 \mathrm{~mL}$ (respectively chromatogram from top to bottom; integration of urea peak at $143.05(\mathrm{~m} / \mathrm{z})$ and $2.86 \mathrm{~min}(\mathrm{Rt}))$. 


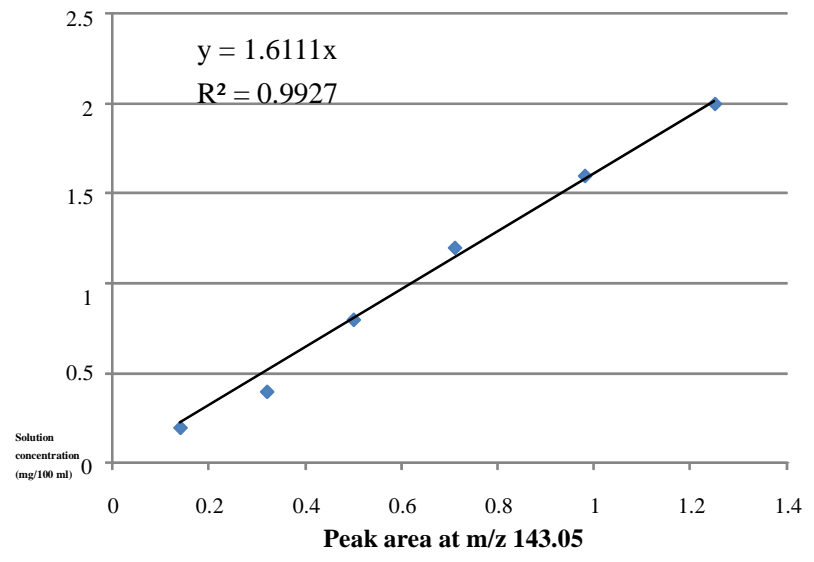

Figure 3. LC/ESI-HRMS urea analysis calibration curve between 0.2 and $2 \mathrm{mg} / 100 \mathrm{~mL}$.

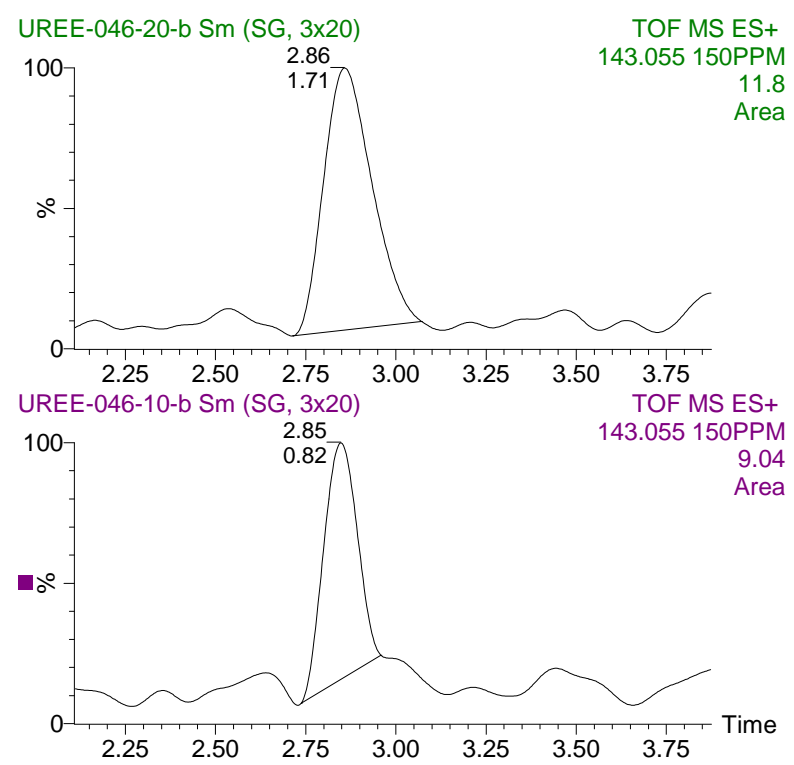

Figure 4. LC/ESI-HRMS urea analysis of dry dog food extracted with a urea solution at $1 \%$ (chromatogram $A$ ) and at $\mathbf{0 . 5 \%}$ (chromatogram B).

The results of this doped samples analyses are shown on Table 6.

The pellet matrix appears to suppress around 35\% the MS signal.

The EC spectro-colorimetric has determined a content of $0.46 \mathrm{~g} / 100 \mathrm{~g}$ in this pet food sample which was doped with urea. In order to take into account the matrix effect on the LC/ESI-HRMS quantitative analysis, the correlation between the urea content in the dry pellets concentration of the doped samples and the LC/ESI-HRMS peak area was established. The extrapolation of the curve to zero added urea gives directly the urea concentration in the analyzed sample (see Figure 5).

It can be determined from the curve the value of 0.05 $\mathrm{g} / 100 \mathrm{~g}$ for the real urea content of this sample. This value is significantly below the EC spectro-colorimetric result
Table 6. Results of analysis by LC/ESI-HRMS (m/z 143.05) of dry pet food pellets doped at $0.5 \%$ and $1 \%$ with pure urea powder.

\begin{tabular}{ccccc}
\hline Sample & $\begin{array}{c}\text { Area m/z } \\
\mathbf{1 4 3 . 0 5}\end{array}$ & $\begin{array}{c}\text { Measured } \\
\text { concentration } \\
\text { (mg/100mL) }\end{array}$ & $\begin{array}{c}\text { Theoretical } \\
\text { concentration } \\
\text { (mg/100mL) }\end{array}$ & $\begin{array}{c}\text { Extraction } \\
\text { yields (\%) }\end{array}$ \\
\hline $\begin{array}{c}\text { Doped at } \\
\mathbf{0 . 5 \%}\end{array}$ & 0.82 & 1.32 & 2 & 66 \\
$\begin{array}{c}\text { Doped at } \\
\mathbf{1 \%}\end{array}$ & 1.71 & 2.75 & 4 & 69 \\
\hline
\end{tabular}

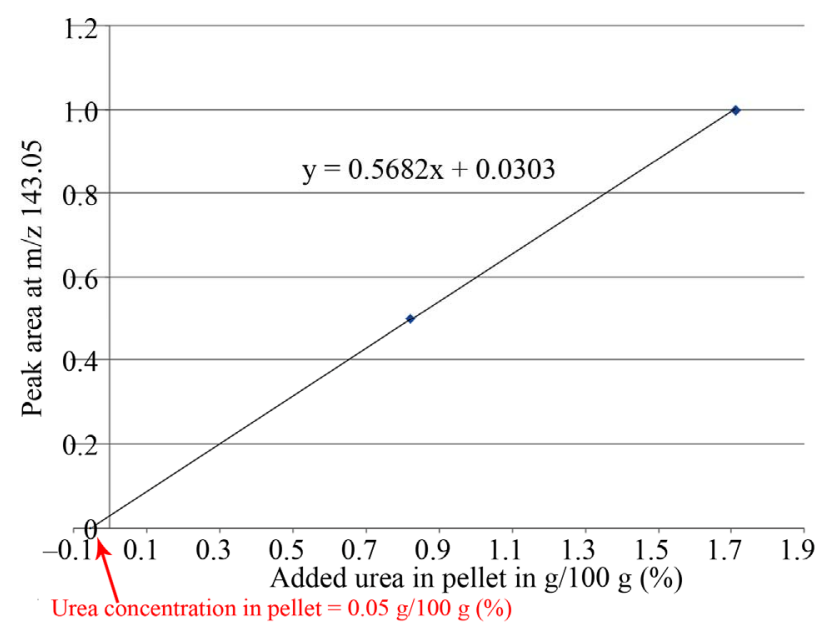

Figure 5. Extrapolation of the correlation between peak area and urea concentration in doped pellet sample (g/100g) to determine the real content of urea in the analyzed sample.

of $0.46 \mathrm{~g} / 100 \mathrm{~g}$. Spiking method, being less accurate than calibration method, further analyses are necessary to validate this result.

\subsection{LC/ESI-HRMS and Spectro-Colorimetric or Enzymatic Methods Comparison}

Further analyses were performed to compare the EC spectro-colorimetric method, the enzymatic method and LC/ESI-HRMS. The selected samples are described in Table 7.

A new calibration file was established and results are shown on Figures 6 and 7.

The chromatograms and peak areas obtained by analysis of samples listed in Table 7 are presented on Figure 8 (the chromatogram at the top corresponds to a standard solution at $0.2 \mathrm{mg} / 100 \mathrm{~mL}$ urea).

The peaks at retention time between 2.70 and $3.05 \mathrm{~min}$. were taken as urea peak to keep the worst case hypothesis. The results for the five analyzed samples are presented on Table 8.

The results obtained by LC/EIS HRMS are significantly below the results obtained by EC colorimetric method and in the same magnitude than the results of the enzymatic analysis $(<0.01 \mathrm{~g} / 100 \mathrm{~g}$, Table 7$)$ even if the corrective factor to take into account the $65 \%$ yield is not applied (see Table 9). 
Table 7. Selection of a range of petfood samples for analysis by LC-ESI-HRMS.

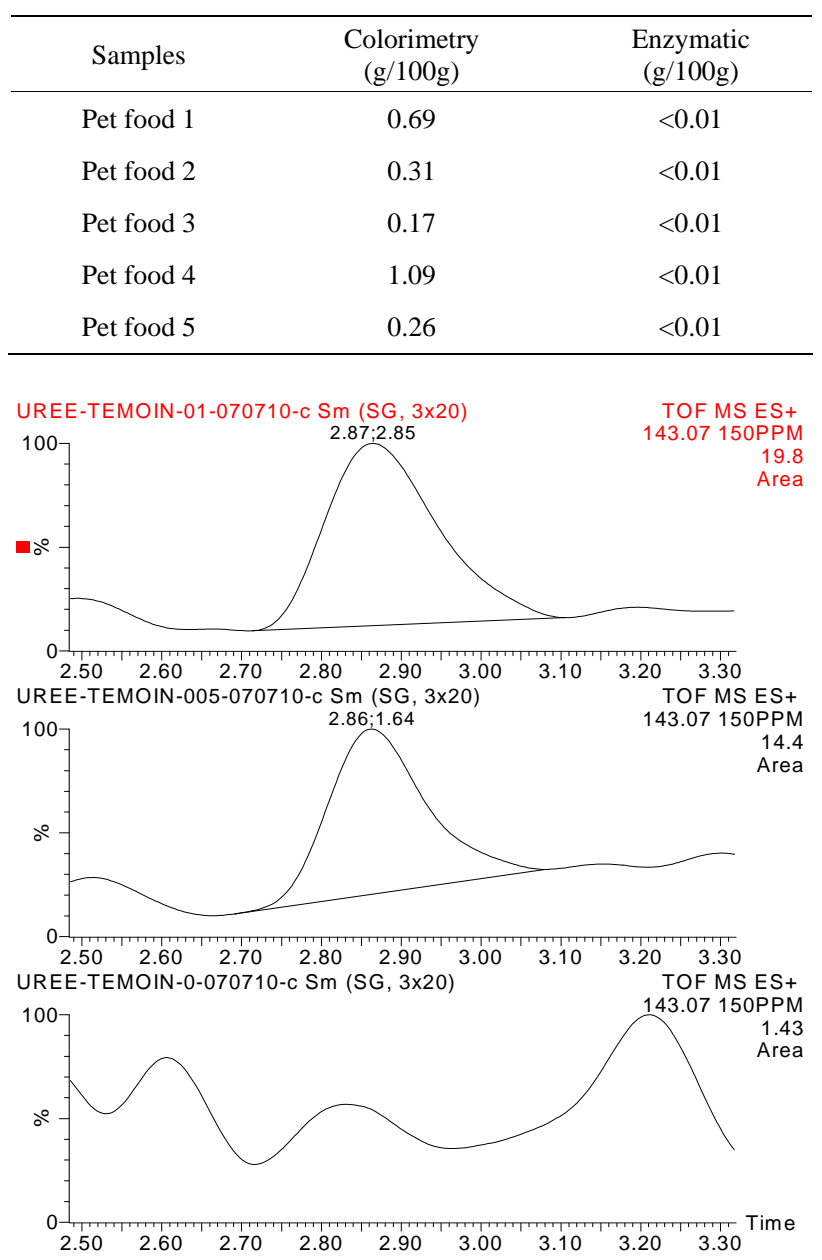

Figure 6. LC/ESI-HRMS analysis of urea standard solution at $0,0.2$ and $0.4 \mathrm{mg} / 100 \mathrm{~mL}$ concentrations (respectively chromatogram from top to bottom; integration of urea peak at $143.07(\mathrm{~m} / \mathrm{z})$ and $2.86 \mathrm{~min}(\mathrm{Rt}))$.

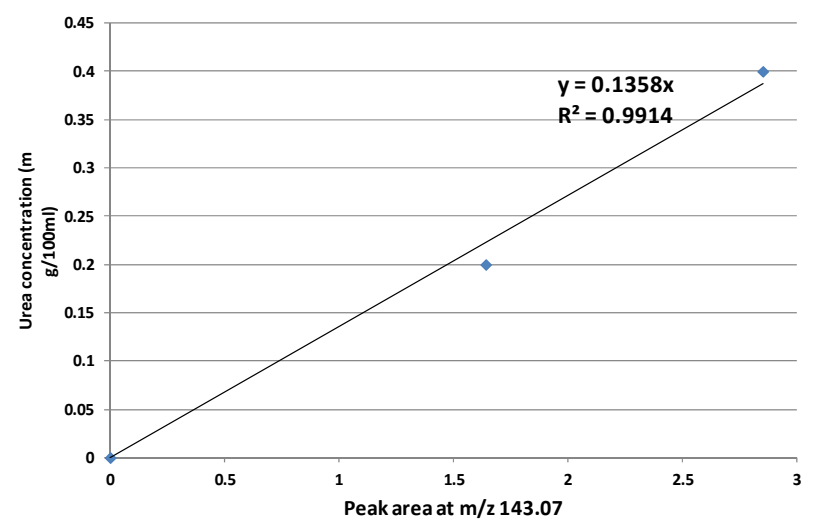

Figure 7. LC/ESI-HRMS urea analysis calibration curve at $0,0.2$ and $0.4 \mathrm{mg} / 100 \mathrm{~mL}$.

The concentrations analyzed by LC/ESI HRMS for this set of dry pet food samples are in the magnitude order of
UREE-TEMOIN-005-070710-c Sm (SG, 3 × 20)
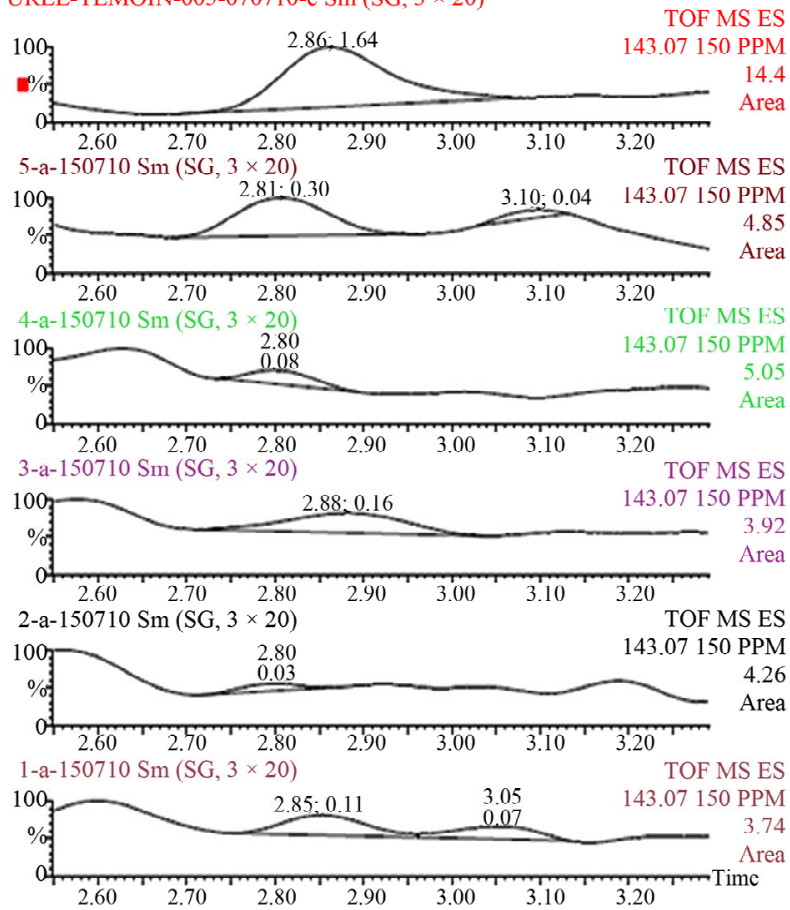

Figure 8. LC/ESI-HRMS urea analysis of dry dog food water extracts. (Pet food 1: chromatogram at the bottom and respectively pet food $2,3,4,5$ from bottom to top of the figure, chromatogram at the top corresponds to a standard solution at $0.2 \mathrm{mg} / 100 \mathrm{~mL}$ urea).

Table 8. Results of analysis of dry pet food samples by LC/ESI-HRMS (m/z 143.07) (2 g of samples extracted in 500 mL of water).

\begin{tabular}{cccc}
\hline & $\begin{array}{c}\text { Peak area m/z } \\
143.04\end{array}$ & $\begin{array}{c}\text { Water extract } \\
\text { concentration }(\mathrm{mg} / 100 \mathrm{~mL})\end{array}$ & $\begin{array}{c}\text { Pellet content } \\
(\mathrm{g} / 100 \mathrm{~g})\end{array}$ \\
\cline { 2 - 4 } $\begin{array}{c}\text { Standard }(0.2 \\
\mathrm{mg} / 100 \mathrm{~mL})\end{array}$ & 1.64 & 0.223 & na \\
\hline Pet food 1 & 0.11 & 0.015 & 0.004 \\
Pet food 2 & 0.03 & 0.004 & 0.001 \\
Pet food 3 & 0.16 & 0.022 & 0.005 \\
Pet food 4 & 0.08 & 0.011 & 0.003 \\
Pet food 5 & 0.3 & 0.041 & 0.010 \\
\hline
\end{tabular}

na: not applicable.

Table 9. Comparison of urea content in dry pet food pellets obtained by EC colorimetric method, enzymatic method and LC/ESI-HRMS.

\begin{tabular}{cccc}
\hline Samples & Colorimetric method Enzymatic method & $\begin{array}{c}\text { LC/ESI-HRMS } \\
\text { method }\end{array}$ \\
\cline { 2 - 4 } & $(\mathrm{g} / 100 \mathrm{~g})$ & $(\mathrm{g} / 100 \mathrm{~g})$ & $(\mathrm{g} / 100 \mathrm{~g})$ \\
\hline Pet food 1 & 0.69 & $<0.01$ & 0.004 \\
Pet food 2 & 0.31 & $<0.01$ & 0.001 \\
Pet food 3 & 0.17 & $<0.01$ & 0.005 \\
Pet food 4 & 1.09 & $<0.01$ & 0.003 \\
Pet food 5 & 0.26 & $<0.01$ & 0.010 \\
\hline
\end{tabular}


the enzymatic method $(<0.01 \%)$ when the EC colorimetric method resulted in urea concentration between $0.1 \%$ to $1 \%$.

\section{Conclusions}

As a conclusion, the EC colorimetric method for urea analysis is not applicable to dry petfood products as interfering molecules result in significant urea concentration over dosing.

The interfering molecules may be free amino-acids as they react with DMBA and absorb at $435 \mathrm{~nm}$ as shown by the LC/UV-DAD analyses. As a matter of fact ingredients known to contain significant level of free amino acid present some content of urea when analyzed by the EC colorimetric method.

The enzymatic method gives results $<0.01 \mathrm{~g} / 100 \mathrm{~g}$ for all petfood samples and is proven to be able to detect urea in petfood samples when urea is spiked and also by analysis of some ingredients like pork liver which may naturally contain urea.

After several trials and studies, the analysis of a range of dry petfood by LC/ESI-HRMS has allowed the validation of the results obtained using the enzymatic method.

The recommendation would be to propose a modification of the EC Commission Regulation No. 152/2009 and to recommend the enzymatic method for monitoring urea in pet food products and in food product with complex matrices and significant level of free amino acids. This would also harmonize the regulation between Europe and America zones as the AOAC already recommends the enzymatic method for urea analysis in pet food.

The complete validation of the LC/ESI-HRMS would require further work to validate the extraction method and to study the stability of extracted petfood water solutions doped with urea. This method is also quite sophisticated and expensive for routine control in analytical laboratories. A promising approach using a benchtop Orbitrap mass spectrometer for mass accuracy improvement (mass selection window less than $3 \mathrm{ppm}$ ) [18] may also be considered.

As a general comment the methods developed and validated for feed are often not suitable to petfood as the two categories of products present very different compositions.

\section{Acknowledgements}

The authors are thankfull to the Eurofins laboratory who have conducted the colorimetric and enzymatic analyses of pet food samples.

\section{REFERENCES}

[1] P. Varelis and R. Jeskelis, "Preparation of [13C3]-Melamine and [13C3]-Cyanuric Acid and Their Application to the Analysis of Melamine and Cyanuric Acid in Meat and
Pet Food Using Liquid Chromatography-Tandem Mass Spectrometry," Food Additives \& Contaminants: Part A, Vol. 25, No. 10, 2008, pp. 1208-1215. doi:10.1080/02652030802101893

[2] K. Ertell, "A Review of Toxicity and Use and Handling Considerations for Guanidine, Guanidine Hydrochloride, and Urea,” Battelle Memorial Institute Pacific Northwest National Laboratory, US Department of Commerce, Springfield, 2006.

[3] F. M. McNabb and R. A. McNabb, "Proportions of Ammonia, Urea, Urate and Total Nitrogen in Avian Urine and Quantitative Methods for Their Analysis on a Single Urine Sample,” Poultry Science, Vol. 54, No. 5, 1975, pp. 1498-1505.

[4] P. A. Wright, "Nitrogen Excretion: Three End Products, Many Physiological Roles,” The Journal of Experimental Biology, Vol. 198, 1995, pp. 273-281.

[5] P. S. Francis, S. W. Lewis and K. F. Lim, "Analytical Methodology for the Determination of Urea: Current Practice and Future Trends,” TrAC Trends in Analytical Chemistry, Vol. 21, No. 5, 2002, pp. 389-400. doi:10.1016/S0165-9936(02)00507-1

[6] Commission Regulation (EC) No. 152/2009 of 27 January 2009, "Laying down the Methods of Sampling and Analysis for the Official Control of Feed," Official Journal of the European Union, 2009.

[7] Association of Official Analytical Chemistry, "Official Method of Analysis of the Association of Official Analytical Chemistry," 12th Edition, Association of Official Analytical Chemistry, Washington DC, 1975, p. 132

[8] Association of Official Analytical Chemistry, “Official Method of Analysis of the Association of Official Analytical chemistry,” 16th Edition, Association of Official Analytical Chemistry, Washington DC, 2002.

[9] W. Kulik, M. J. S. Oosterveld, R. M. Koka and K. de Meera , "Determination of $13 \mathrm{C}$ and 15N Enrichments of Urea in Plasma by Gas Chromatography-Combustion Isotope Ratio Mass Spectrometry and Gas Chromatography-Mass Spectrometry Using the 2-Methoxypyrimidine Derivative,” Journal of Chromatography B, Vol. 791, No. 1-2, 2003, pp. 399-405.

[10] A. Kessler and L. Siekmann , "Measurement of urea in human serum by isotope dilution mass spectrometry: A reference procedure,” Clinical Chemistry, Vol. 45, No. 9, 1999, pp. 1523-1529.

[11] X. Dai, X. Fang, F. Su, M. Yan, H. Li, J. Zhou and R. Xu, "Accurate Analysis of Urea in Milk and Milk Powder by Isotope Dilution Gas Chromatography-Mass Spectrometry," Journal of Chromatography B, Vol. 878, No. 19, 2010, pp. 1634-1638. doi:10.1016/j.jchromb.2010.04.005

[12] R. A. Kohn, K. R. French and E. Russek-Cohen, “A Comparison of Instruments and Laboratories Used to Measure Milk Urea Nitrogen in Bulk-Tank Milk Samples,” Journal of Dairy Science, Vol. 87, No. 6, 2004, pp. 1848-1853.

[13] M. M. Hojjatie, D. E. Abrams and T. M. Parham (Jr.), "Liquid Chromatographic Determination in Water Soluble Urea Formaldehyde Fertilizer Products and in Aqueous Urea Solutions: Collaborative Study,” Journal of 
AOAC International, Vol. 87, No. 2, 2004, pp. 346-351.

[14] M. Koebel and M. Elsener, "Determination of Urea and Its Thermal Decomposition Products by High-Performance Liquid Chromatography," Journal of Chromatography $A$, Vol. 689, No. 1, 1995, pp. 164-169. doi:10.1016/0021-9673(94)00922-V

[15] Macherey Nagel Application Note 120440, “Separation of Urea, Biuret and Cyanuric Acid (Courtesy of C. Greve)," University of Clausthal, Institute of Chemical Engineering, Clausthal, 2005.

[16] H. Abidi, J. L. Gass and M. F. Grenier-Loustalot, "Analyse Quantitative de l'Urée dans l'Eau par HPLC-APCIMS-MS et HPLC-ES-MS-MS," L'Actualité Chimique, No. 4, 2001, pp. 33-36.
[17] H. R. Morris, T. Paxton, A. Dell, J. Langhorne, M. Berg, R. S. Bordoli, J. Hoyes and R. H Bateman, "High Sensitivity Collisionally-Activated Decomposition Tandem Mass Spectrometry on a Novel Quadrupole/OrthogonalAcceleration Time-of-Flight Mass Spectrometer," Rapid Communications in Mass Spectrometry, Vol. 10, No. 8, 1996, pp. 889-896.

[18] J. V. Olsen, L. M. F. de Godoy, G. Li, B. Macek, P. Mortensen, R. Pesch, A. Makarov, O. Lange, S. Horning and M. Mann, "Parts per Million Mass Accuracy on an Orbitrap Mass Spectrometer via Lock Mass Injection into a C-Trap,” Molecular \& Cellular Proteomics, Vol. 4, No. 12, 2005, pp. 2010-2021. 